\title{
Primary fibromatosis of the breast in a 13 years old girl
}

\author{
Elhassani Mehdi ${ }^{1}$, Kouach Jaouad ${ }^{2 *}$, Achenani Mouna ${ }^{2}$, Babahabib Abdellah ${ }^{2}$, Moussaoui Driss ${ }^{2}$, \\ Dehayni Mohamed ${ }^{2}$, Bakkali Hicham ${ }^{3}$ \\ ${ }^{1}$ Gynecology-Obstetrics Department, ElRapaha Polyclinic, Libreville, Gabon \\ ${ }^{2}$ Gynecology-Obstetrics Department, Mohamed V Military Hospital for Instruction, Rabat, Morocco \\ ${ }^{3}$ Anesthesiology Department, ElRapaha Polyclinic, Libreville, Gabon \\ Email: ${ }^{k}$ kouach_jaouad@yahoo.fr
}

Received 13 May 2012; revised 20 June 2012; accepted 5 July 2012

\begin{abstract}
The mammary fibromatosis is a rare clinical entity. The etiopathogeny remains unclear. The clinical and radiological characteristics of this injury are often worrying within the main differential diagnosis of the mammary carcinoma; however only the histological examination can confirm the diagnosis. It is characterized by a strictly local evolution with an infiltration and recidivism tendency without giving metastasis. The treatment is basically surgical. We report herewith an observed case of 13-year-old girl, presenting with a huge tumor located in the left breast with ulceration of the nipple-areolar plate. The diagnosis is confirmed by the histological study. The treatment consisted of a mastectomy. Suites are simple. In view of the clinical evidence of the literature, we call back the difficulties of management of this tumor.
\end{abstract}

Keywords: Breast; Fibromatosis; Desmoid Tumor; Extraabdominal Desmoid Tumors; Gardner's Syndrome

\section{INTRODUCTION}

The agressive fibromatosis or desmoid tumor is a mesenchymal tumor that corresponds to à fibroblastic proliferation, having an intermediate biological behavior between the benign fibroblastic lesions and the low grade fibrosarcoma. It tends to recur, but with no metastas anymore. According to its location, we distinguish the abdominal fibromatosis (25\% - 49\%), the extra-abdominal fibromatosis $(43 \%-60 \%)$ as well as the intraabdominal fibromatosis (8\% - 15\%) [1,2].

The mammary location represents only a particular aspect of these tumors, its frequency is less than $0.2 \%$ of the primitive tumors of the breast and $4 \%$ of the extraabdominal fibromatosis. She can arise from the fascia musculo-aponeurotic of the chest wall or from fibroblasts and myofibroblasts of the mammary parenchyma [1-3].

*Corresponding author.
We report herewith a new case of desmoid tumor in a 13-year-old teenager, and In view of evidence of the literature, we focus on the clinical aspects, the diagnostic and therapeutic difficulties of this tumor.

\section{OBSERVATION}

Miss P.K., 13-year-old, of Gabonese origin, having a regular cycle for two years, she is being admitted to the department for a large ulcerated tumor by the left breast. Her personal and family histories were without peculiarity in particular no breast cancer. The beginning of the disease goes back up to one year by a self-discovery of a tumor by the left breast having quickly increased in volume with ulceration of all the nipple-areolar plate. All this evolved in preservation context of the general state. The examination finds the left breast increased in volume with palpation of a mobile firm mass with regard to the profound plan, the skin with regard to the nipple-areolar plate is hurt with purulente serosity, covering the whole breast and being $15 \mathrm{~cm}$ of diameter (Figure 1). The examination of the breast contralateral is without peculiarity. The palpation of the ganglionic areas is without abnor-

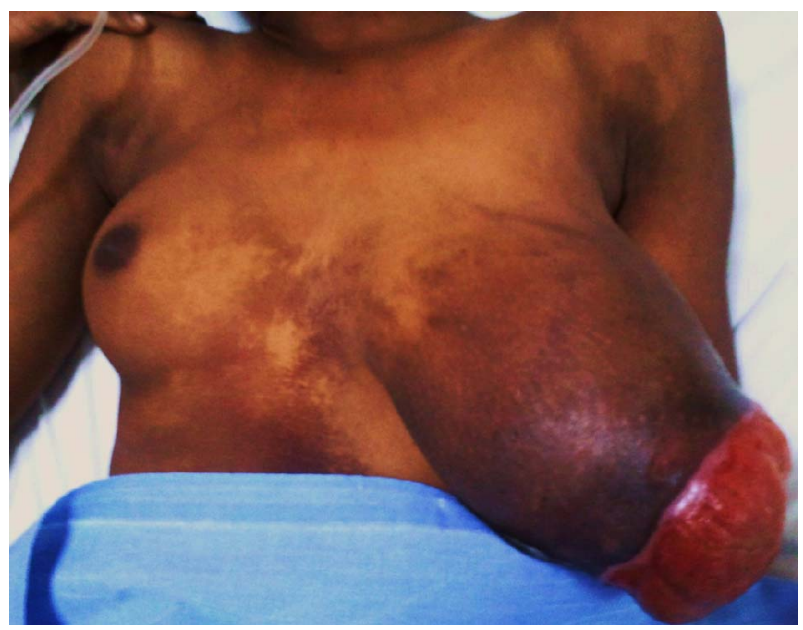

Figure 1. Large tumor of the left breast with ulceration of the nipple-areolar plate. 
malities. The mammography was not able to be achieved and the echography showed a heterogeneous tissular mass of which the big diameter $>14 \mathrm{~cm}$. The cytoponction been not decisive, biopsies were realized. The histological examination noticed a proliferation of well differentiated regular fibroblasts, in the nucleus (kernel) sometimes dystrophic with rare normal mitoses, the fibroblastic tissue was arranged in beams in support of a desmoid tumor of the left breast (Figure 2). Given the tumour size and the risk of a second recurrence, the patient benefited from a left breast mastectomy. The histological study of this part confirmed the diagnosis of desmoid tumor with healthy limits. The post-operative evolution was favorable. The clinical supervision did not show local second recurrence with a recession of 6 months. A mammary plasty is expected after 03 years of evolution without a local recurrence.

\section{DISCUSSION}

The mammary fibromatosis is a rare lesion, often described as isolated cases or small series.

It represents $0.2 \%$ of all the tumors of the breast $[1,2]$. It primarily affects women from 13 to 80 years old with an average of 43 years, regardless of their ethnicity [2,3], unlike the fibromatosis of the abdominal wall, the mammary location is not mainly associated with the adolescence. The mammary fibromatosis is more commun in women than in men [2-4].

The etiopathogeny of desmoid tumors is not clear. It may be the result of three factors: a cell proliferation enhancing, possibly further to a trauma, a developing sexual steroids and a particular genetic conditions affected by a regulation problem of the fibroblast growth $[1,3,4]$.

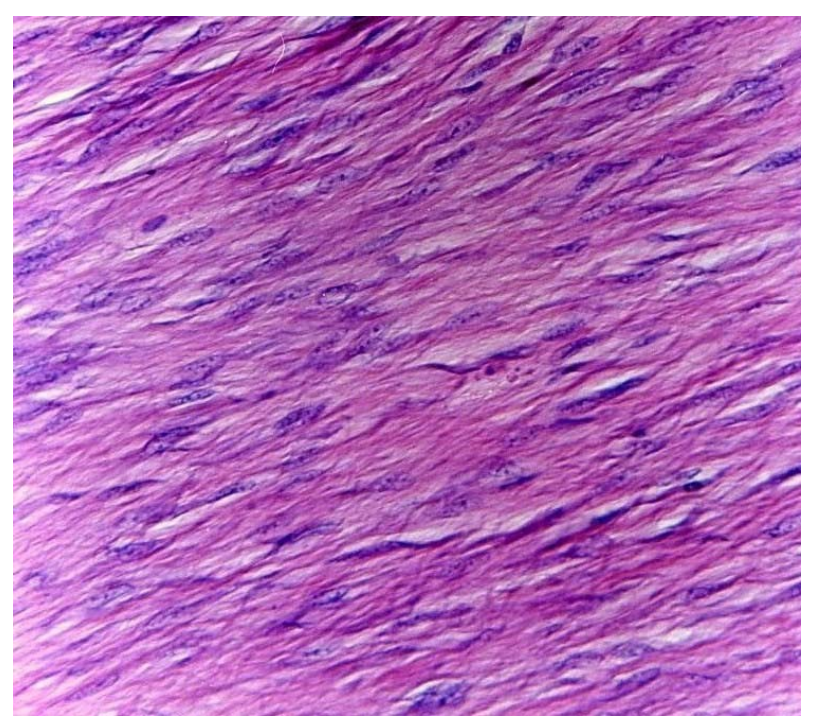

Figure 2. Regular spindle-shaped cells, without atypia.
The clinical aspect is mostly about one-sided and firm mammary gland nodule, generally painless, but with fast and alarming increase, indistinct and irregular limits, variable sizes $(1-10 \mathrm{~cm})$, affecting predominantly the areal area of the right breast, sometimes with a nipple discharge and unusual skin retraction $[2,4]$. Adenopathy is usually absent [1,3-5].

The mammography is usually to have a bad opaqueness limited to fuzzy and irregular edges, architectural distortion area with convergence effect and/or fibrous divergence; a focal asymmetry of density suggestive of malignant lesion but typically without microcalcifications. We sometimes observe a thickening or a skin retraction [1,3,5-7]. The literature reports other alternative descriptions so unusual. The lesion sometimes appears as a mass with a lobulated or not outlines, but still well defined [4-8]. An ossifying lesion was also noticed. The associated microcalcifications are rare. Finally, in some rare cases, the lesion has no mammographic presence [6-8].

The echographic appearances are various and less specific. They may include a hypo-échogènic lesion in the irregular outlines, in the main vertical line with or without post-mitigation [3,6-8]. The mammary fibromatosis can also appear in an area with irregular and/or ill-defined outlines, heterogeneous, hyper outlines and hypoechogenic with or without post-mitigation [6-8]. The literature have also described the small-sized lesions that are homogeneous lesions in very regular outlines $[7,8]$.

Authors suggest the contribution of the MRI in the preoperative diagnosis of fibromatosis mammary. In T1weighting, the lesion is isosignal in the fibro-glandular tissue. In T2-weighting, with suppression of the fat signal, we have a lesion presenting areas of low signal (collagen tissue) and of high signal (myxoid tissue). After the injection of chelated forms of gadolinium, in T1-weighting, and after a fat signal saturation, we notice a heterogeneous raising in the ill-defined outlines. In dynamic acquisition, the lesion presents a progressive raising, thing which is not typical to the mammary carcinoma $[3,4,7,8]$. The magnetic resonance imaging and the scanner are importante to estimate a possible parietal invasion [4, $7,8]$.

The cytoponction with the fine needle could confirm the diagnosis by isolating fibroblasts with minimal atypia; however, it is mostly little contributory [1-4].

The diagnosis certainty is based upon the post surgical histopathology.

Macroscopically the legions are fibrous, brilliant, greyish-white, firm, infiltrating, variable-sized and badly limited [2,3,5,6].

The histological examination showed a proliferation of spindle-shaped cells, fibroblastics and myofibroblastics, with a variable cellularity, minimal atypia and a weak 
mitotic activity, arranged in interlacing bundles which infiltrate the fatty tissue, mixed (involved) with collagen bands. A lymphoplasmacytic infiltration is mostly associated, unlike the calcifications and the oedema that are usually absent [1,3,5,6,9].

In the surrounding area, the lesion is badly limited and infiltrates the mammary parenchyma with extensions in sensor pocket. The cellularity is more abundant in young woman and the hormonal receptors; the epithelials are usually absent.

The differential diagnosis problem arises in a different way depending on the cellularity degree [3,5,7].

In the weak cellularity, cases to be mentioned are:

- The nodular fascist generally too deep, well bounded, with rapid growth and including an inflammatory infiltrate usually non-existent in the fibromatosis;

- The fat necrosis coming along with many foamy histiocytes and sometimes with a granulomatous epithelioid around necrosed fat cells;

- The three diagnosis that have been of a great help to the clinical history; the keloid or the hypertrophic scarring; the traumatic and post-surgical lesions; the fibrosis (radique).

In the increased cellularity cases, the malignancies to be mentioned are:

- The metaplastic carcinoma with spindle-shaped cells showing more (pléio) morphism and many mitoses. Besides, cells are positive keratin;

- Sarcomatous tumors (fibrosarcoma of low grade, malignant (fibrohistiocytome) showing a very important (pléio) morphism, many mitoses of necrosis and a possible vascular spread;

- Liposarcomas, generally with a big size, including stretched or cystic epithelial elements, in or near a strengthening of the cellularity.

The utility of a systematic research for other locations does not make the unanimity. Mostly sporadic, the fibromatosis is associated in $2 \%$ of the cases with familial adenomatous polyposis (FAP) which is the consequence of a transformation of the APC gene (family adenomatous polyposis coli). Some authors recommend to realize, as principle, a colonoscopy to eliminate the rare combination with a polypose in Gardner's syndrome [3-5,8,9].

The mammary fibromatosis is a locally aggressive tumor, but without metastatic potential. The curative surgical resection remains the treatment of choice in all the publications. All authors agree on the need of a wide and radical surgical treatment moving to healthy areas of at least $1 \mathrm{~cm}$ so as to limit the risk of recurrences. If edges are invaded, a revision surgery should be performed. Most of recurrences show up in the first three years which justifies a clinical and radiological medium-term follow-up $[1,3,4,8,9]$.

The literature reports rates recurrence to be from $5 \%$ to $25 \%$, most within three to six years after the excision sometimes more than 10 years later. It seems appropriate to suggest a new curative surgical resection in case of recurrence. The mastectomy can be indicated for vast fibromatosis or for too large recurrences [2-4,8-10]. The node dissection has no indication in this pathology. In case of parietal invasion, the surgery can be extremely decaying going as far as taking away the breast, the pectoral muscles, the thoracic wall, and the parietal pleura. Because of the recurrence risk, a reconstructive breast surgery should be performed ideally after 3 years [1, 8-10].

The place of radiotherapy is being discussed. The results of the various series being often discordant. It requires a strong doses to be effective almost superior to 60 Gy. Some made assumptions of a malignant transformation possibility of the fibromatosis after radiotherapy associated with the surgery. Nevertheless, other studies have shown a beneficial effect of the radiotherapy with $60 \%$ of decreasing cases non-accessible to the surgery. Therefore, the radiotherapy could be curative in recurrence cases when the surgery is impracticable $[4,6,7$, $9,10]$.

Several authors propose an alternative chemotherapy to an aggressive surgery that can leadto serious aftereffects. The treatment by anti-estrogen (tamoxifen), in spite of some described answers, does not seem to be justified because of the absence of hormone receptors $[1,3,6,9]$.

The use of progestin came along with an increasing size of a mammary tumor $[2,3,5,9]$.

\section{CONCLUSION}

The mammary fibromatosis is a benign and infiltrated rare tumor. Its clinical and radiological aspect is suggestive of malignant tumor. Only histology will bring the diagnosis. Its étiopathogeny remains controversial. Its treatment consists of a complete exereses with healthy safety margins so as to reduce recurrence risk. The area of the radiotherapy and the medical treatments, especially anti-oestrogens, are not clearly defined, and limited to this location. The realization of a colonoscopy in principle because of its possible association with the polypose in frame of Gardner's syndrome.

\section{REFERENCES}

[1] Croce, S., Letourneux, C. and Dale, G. (2009) The mammary fibromatosis: A benign lesion relatively unknown. Gynécology-Obstétric \& Fertility, 37, 442-446.

[2] Roman, H., Caron, P., Verspyck, E., et al. (2001) Fibromatose primitive du sein. Primary fibromatosis of the breast. Annales de Chirurgie, 126, 561-564. 


\section{doi:10.1016/S0003-3944(01)00551-X}

[3] Corbisier, C., Garbin, O.D., Jacob, D., et al. (1997) Rare breast tumor: The mammary fibromatosis report of two cases and the literature review. Journal De Gynecologie Obstetrique et Biologie de la Reproduction, 26, 315-320.

[4] Aubertin, A., Anglade, E.G. and Bertrand, G. (2004) The mammary fibromatosis: Report of 5 cases and the literature review. Le Sein, 14, 269-278.

[5] Muller, M., Dessogne, P., Baron, M., et al. (2011) The mammary fibromatosis in a 9-years-old girl. Annals of Pathology, 31, 41-45. doi:10.1016/j.annpat.2010.09.011

[6] El Demellawy, D., Herath, C., Khalil, M., et al. (2011) Fibromatosis of the male breast with concurrent floridtype gynecomastia: Report of a case and review of literature. Pathology—Research and Practice, 207, 306-309. doi:10.1016/j.prp.2010.11.005
[7] Cornford, E.J., Poller, D.N., Ellis, O., et al. (1993) The mammographic appearances of fibromatosis of the breast. The Breast, 2, 175-179. doi:10.1016/0960-9776(93)90063-L

[8] Mesurolle, B., Ariche-Cohen, M., Mignon, F., et al. (2001) Unusual mammographic and ultrasonographic findings in fibromatosis of the breast. European Radiology, 11, 22412243. doi: $10.1007 / \mathrm{s} 003300100956$

[9] Thomas, V.T. and Jose, S. (2011) Bilateral breast fibromatosis: Case report and review of the literature. Journal of Surgical Education, 68, 320-325. doi:10.1016/j.jsurg.2011.02.001

[10] Pang, L.M., Tse, G.M.K., Bonita, K.B. and Law, B.K.B. (2003) MR imaging of primary breast fibromatosis. European Journal of Radiology Extra, 46, 101-104. doi:10.1016/S1571-4675(03)00057-9 